\title{
Die Schallplatte als kommunikatives Medium
}

\author{
von Gottfried Bitter
}

Einem medienpolitischen Anachronismus scheint es gleichzukommen, wenn nach der "zweiten großen Revolution nach Gutenberg“ (M. Mc Luhan), dem Eintritt in das gegenwärtige audiovisuelle Zeitalter, noch ausführlicher über (und für?) das Medium Schallplatte gesprochen wird. Hoffnungslos hinterwäldlerisch muß es sich ausnehmen, wenn hier über die Schallplatte als Kommunikationsmedium gehandelt wird, erwartet man doch in diesen Tagen den serienmäßigen Vertrieb der Video-Platte. Mit dem bloßen Hinweis auf die kommerzielle Bedeutung des Schallplattenmarktes - sein rasches Ende wurde bei der wachsenden Bedeutung des Tonbandes prophezeit; im Jahr 1970/71 wurden etwa 700 Millionen Singles und LPs in der BRD verkauft ist die kommunikative Funktion noch nicht geklärt. Vielmehr muß in einer allgemeinen Diskussion der Medienbedeutung in Lernprozessen (1) die spezielle Funktion der auditiven Medien, insonderheit der Schallplatte, erörtert werden (2), um ihren kommunikativen Rang und ihre Verwendbarkeit in multimedialen Systemen beurteilen zu können (3). ${ }^{1}$

\section{Die Bedeutung der Medien im Lernprozeß}

Als am Ende der 60er Jahre in den Erziehungswissenschaften die didaktischen Fragestellungen in einer bisher nicht gekannten Intensität nach vorne treten, setzt eine umfassende Analyse und Theoriebildung aller an Lernprozessen beteiligten Faktoren ein. Die Didaktik als "Theorie der Bildungsinhalte und des Lehrplans" — so ein Buchtitel E. Wenigers von 1930 - wird weiterentwickelt zur "Theorie optimalen Lehrens und Lernens" - vor allem von S. B. Robinsohn und seiner Berliner Arbeitsgruppe. Die Gesamtheit der Überlegungen eines systematisierten Plans der Lernziele und Lehrinhalte (Lehrplan) zusammen mit den zu entwickelnden Lehrmitteln bzw. Medien (Lehrbücher, Arbeitshefte, Filme, Schallplatten, Tonbänder u. ä.) und entsprechenden Lernkontrollen - Curriculum genannt ${ }^{2}$ - wird zum bevorzugten Forschungsgegenstand. Das Curriculum will Lernprozesse anregen, steuern und zu bestimmten Zielen (Qualifikationen) bringen.

Nach der Bedeutung der Medien fragt die Curriculumforschung innerhalb der Untersuchung der Bauprinzipien neuer Curricula und ihrer ständigen Revision; dabei wird als kaum zu überschätzende Nebenwirkung für die Medienfrage Dreierlei hervorgehoben: die sorgfältige Beobachtung der Interdependenzen zwischen den Curriculumelementen (Lernziele, Lerninhalte, Lernverfahren) und die Rückkopplung von Theorie und Praxis in der Entwicklung, Durchführung und Evaluierung der Curricula kommt einer intensiveren Medienplanung, einer erhöhten Verwendung und einer gezielteren Wirkungskontrolle der Medien in den Lernprozessen zugute; schließlich wird der Ort der Medienplanung innerhalb der Organisation der Lernprozesse deutlicher erkannt.

P. Gottfried Bitter CSSp ist wissenschaftlicher Assistent und Doktorand am Seminar für Pastoraltheologie im Fachbereich Katholische Theologie der Westfälischen Wilhelms-Universität Münster. Er betreute für den Christophorus-Verlag (Herder Freiburg) die Redaktion einer Sprechplattenserie für die theologische Erwachsenenbildung. 
Außerdem treten offener als bisher zwei Leerstellen in der Medienpraxis zutage: das Fehlen einer allgemeinen Medienpraxis ${ }^{3}$ und einer empirisch angelegten Forschung der Medienwirkungen. ${ }^{4}$ Kurz: Durch die Curriculum-Arbeit mit ihrem hohen Methodeninteresse sind die Medien, d. h. alle gegenständlichen Lehr- und Lernmittel, vom Arbeitstext bis zum Sprachlabor, merklich in den Bereich pädagogischer Forschung gerückt worden. Hinweis für das zunehmende Medieninteresse ist das wachsende Angebot von (curricularen) Unterrichtsmodellen zusammen mit Medienpaketen $^{5}$ ähnlich wie die Lehrmittelausstellungen (bes. Didacta 1970 und 1972 in Basel und Hannover) und AV-Messen (z. B. VIDCA in Cannes 1972). ${ }^{6}$ Inzwischen werden auch neben den schon lange üblichen allgemeinen Medienkatalogen (meist geordnet nach Mediengattungen: visuelle, akustische, audiovisuelle Lehrmittel) spezielle Mediensammlungen angeboten, eigens zusammengestellt für ein bestimmtes Lehrbuch bzw. Lernprogramm. ${ }^{7}$

Auffällig bei diesen erfreulichen Lernmittelangeboten ist vielfach das mangelnde Geschick beim Einsetzen der Medien von seiten der Autoren; hier wirken sich offensichtlich die Mängel einer fehlenden allgemeinen Mediendidaktik ähnlich aus wie die geringe Bedeutung, die bislang die Lernpsychologie dem Lernen mit Medien schenkte. ${ }^{8}$ Vor allem das Korrelieren von Medieneinsatz und Sozial- und Aktionsformen im Lernprozeß wird kaum beachtet. ${ }^{9}$ Die Folge dieser Mängel ist bei vielen Lehrpersonen in Schule und Erwachsenenbildung ein Medienüberdruß, denn sie stellen fest: die Medienwahl zahlreicher Projekte und Modelle scheint beliebig zu sein, die einzelnen Lehr- und Lernmittel sind in die methodischen Einzelschritte nicht integriert. Kaum daß die Medien stärker in Kommunikations- und Lernabläufe aufgenommen werden, verlieren sie teilweise (schon wieder) an Bedeutung, weil die nintentionale Polyvalenz" (W. Schulz) vieler Medien nicht beachtet wird.

\section{Die Schallplatte als kommunikatives Medium in Schule und Erwachsenenbildung}

Angestoßen durch die Didaktikdiskussion, verstärkt sich das "Medienbewußtsein“ zunehmend; hier wird nun versucht, die spezielle Funktion der auditiven Medien"*, besonders der Schallplatte, innerhalb verschiedener Lernprozesse in Schule und Erwachsenenbildung zu erörtern, und zwar in der Form einer knappen Bilanz (a) und einer Aufzählung der medienspezifischen Vor- und Nachteile der Schallplatte (b).

a) Innerhalb der Methodik der Lernprozesse (im engeren Sinn) wird neben den beiden Bedingungsfeldern (anthropogene und soziokulturelle Voraussetzungen) die Medienwahl zusammen mit den Entscheidungen über die Intentionen und die Sozialund Aktionsformen des Lernprozesses geplant. Nun ist zu beobachten, daß die Schallplatte in Schule und Erwachsenenbildung fast nur in zwei Intentionen eingesetzt wird: nämlich zur Motivierung und zur Rationalisierung, d.h. um im Lernenden eine Bereitschaft zu erwirken, sich mit einem Problem zu befassen und dieses Problem in authentischer Weise zu dokumentieren. Damit sind in etwa schon die beiden kommunikativen Funktionen der Schallplatte in der bisherigen Praxis benannt: eine affektive und kommunitive Funktion, nämlich die Vermittlung des Einstiegs und der Einsicht.10

Diese Beschränkung des Schallplattenmediums auf die genannten beiden Funktionen ist weniger der Einfallslosigkeit der Methodiker ${ }^{11}$ anzulasten als vielmehr großteils die Folge einer einseitig orientierten Schallplattenproduktion für den Schul- und 
Erwachsenenbereich. So werden auf dem Sektor ,Sprechplatten' neben Fremdsprachenplatten und literarischen Programmen (Dichterlesungen, Theatermitschnitten, Kabarett) nur Hörbilder und (historische) Dokumentationen angeboten. ${ }^{12}$ Das Hörbild, vielfach eine Nachbildung von Hörfunkformen (Hörspiel, Reportage, Feature), scheint zugunsten der Tonbildreihe (Diaphonie, Diareihe mit Tonband oder Schallplatte) an Bedeutung zu verlieren. Die historische akustische Dokumentation wird nur noch für einzelne politische, literarische und kirchliche Themen angeboten. ${ }^{13}$ Damit sind einfach vom Schallplattenangebot her die Einsatzmöglichkeiten in Lernabläufen sehr beschränkt.

Ende der 60er Jahre werden mit Schallplatten einige neue Versuche unternommen, die gezielter als bisher die Schallplatte als "didaktisches Medium“ (E. Meyer) in schulische und erwachsenenbildnerische (theologische) Lernprozesse bringen wollen: die Beschränkung auf die beiden kommunikativen Funktionen Einstieg und Einsicht sollen durchbrochen werden, die Schallplatte soll die Einsatzmöglichkeiten eines Lebrund Lernmittels ${ }^{14}$ übernehmen. ${ }^{15}$ Die Formen des Vortrags, des freien und gestellten Dialogs, des Interviews und der Diskussionsrunde werden nach didaktischen Gesichtspunkten eingesetzt, um somit über die Intentionen der Motivierung und Rationalisierung hinaus auch eine Intensivierung und Okonomisierung der Lernprozesse zu erreichen. In eigens für diese Serien konzipierten Beiheften wird neben dem vollen Text der Sprechplatte in einer didaktischen Analyse die Thematik in den Fragehorizont des potentiellen Hörers gestellt, die Bedeutsamkeit des Themas für den Hörer im Augenblick und für die Zukunft erörtert und die Eigengesetzlichkeit des behandelten Gegenstandes bedacht; methodische Uberlegungen über die mögliche Gliederung eines Lernverfahrens anhand einer bestimmten Schallplattenreihe, über Sozialund Aktionsformen und ergänzende Medien samt einer knappen Bibliographie sind angefügt. Jedoch diese Experimentreihen, so darf man wohl vorsichtig sagen, haben nicht den erwarteten Erfolg innerhalb der Schule und Erwachsenenbildung gehabt. Als mögliche Erklärung kann man auf die allgemeine "Medienreserve“16 hinweisen, desgleichen auf die noch zu geringe Beachtung didaktisch-methodischer Fragen in der (theologischen) Erwachsenenbildung ${ }^{17}$ und nicht zuletzt auf das verhältnismäßig geringe Prestige des Mediums Schallplatte für Kommunikations- und Lernprozesse. ${ }^{18}$ Ist aber mit dieser vorab negativen Bilanz die Schallplatte schon als kommunikatives und didaktisches Medium abgetan?

b) Wahrscheinlich ist u. a. die geringe Verwendung der Schallplatte in den verschiedenen Lernprozessen auf die mangelnde Kenntnis allgemeiner und medienspezifischer Vor- und Nachteile der Schallplatte zurüickzuführen. Mit vielen Medien gemeinsam hat die Schallplatte die folgenden fünf allgemeinen Vorteile ${ }^{10}$ :

1) die Objektivierung einer Mitteilung durch das Medium Schallplatte steigert den Rang der Kompetenz des Mitgeteilten;

2) die formale Perfektion der Mitteilung gibt dem Inhalt ein zusätzliches Gewicht;

3) die leichte Reproduzierbarkeit kann Lehrprozessen zugute kommen;

4) der Multiplikationseffekt: „mit Hilfe der Medien können die pädagogischen Prozesse mehr Menschen erreichen und mehr Menschen können an ihnen teilnehmen "20;

5) durch die genannten Vorteile kann der Mitteilungsträger Schallplatte eine Intensivierung der Kommunikations- und Lernprozesse ${ }^{21}$ erzielen. 
Außerdem sind für die Schallplatte noch drei medienspezifische Vorteile zu nennen:

1) Die Schallplatte als „einsinniger“, monomedialer Informationsträger hat eine hohe Affinität zur auslaufenden Wort- und Buchkultur; als Medium des Übergangs vom auditiv-skriptiven zum optischen bzw. audiovisuellen Zeitalter kann die Schallplatte über das Hören-Lernen hinaus das Hören-Sehen-Lernen anbahnen.

2) Die "Bildlosigkeit" des auditiven Mediums Schallplatte wird meist als Nachteil angeführt; stimuliert aber nicht gerade die Bildlosigkeit die Imagination, die Bebilderung des Gehörten im Hörer? Oder umgekehrt: Vermindert u. U. nicht das faszinierende Bild die kritische Distanz? Zumindest kann behauptet werden, daß die auditiven Medien die imaginativen und kreativen Kräfte aktivieren.22

3) Endlich kommt der Schallplatte als einsinnigem, nonvisuellem Medium eine medienkritische Funktion zu - vorab in Sachen Fernsehkonsum. Die Schallplatte kann zu einem kontrollierten Umgang mit audiovisuellen Medien anleiten. Denn gegen ein wachsendes Passiv-Werden durch eine vorgefertigte Bebilderung der Erlebniswelt lehnen sich gerade jene Medien auf, die die Vorstellungskraft der Hörer anspornen. ${ }^{23}$

Die allgemeinen und medienspezifischen Nacbteile der Schallplatte sind meist die Kehrseiten ihrer Vorteile. So bringen die Objektivierung und Perfektion zwangsläufig eine Distanzierung mit sich; die Multiplizierbarkeit hat oft den teilweisen Verlust der Individualität der Lernprozesse zur Folge. Doch die Hauptschwierigkeiten für den breiteren Einsatz von Schallplatten in Kommunikations- und Bildungsprozessen auf verschiedenen Ebenen liegen wohl - abgesehen von der Angewiesenheit auf den Schallplattenmarkt und von der geringen Handhabbarkeit verglichen mit dem Tonband - in ihrer Einsinnigkeit. Das Fehlen des Bildes wird vorab als Mangel gewertet. Der Trainer oder Lehrer muß vielfach erst für das bildlose Medium werben, um das Medienprestige der Schallplatte zu heben. Um diesem Handicap zu entgehen, greift man lieber gleich zum Kurzfilm oder zum Videorecorder.

\section{Die Schallplatte im Medienverbund}

Es ist nicht damit getan, ergänzend (oder sogar als Voraussetzung!) zur "visuellen Bildung“24 eine "auditive Bildung" zu fordern, sondern gründlicher als bisher die allgemeinen und medienspezifischen Chancen der Schallplatte in Lernprozessen zu nutzen, und $z$ war nicht als Einzelmedium, sondern in einem organisierten Medienverbund.

Auf die allgemeine Bedeutung der Medienplanung innerhalb der Vorbereitungsphase von Lernprozessen wurde schon hingewiesen. ${ }^{25}$ Hier ist nun zu fragen: $\mathrm{Zu}$ welchen anderen Medien - unter Einschluß der thema- und teilnehmerbedingten UUberlegungen - bestehen von seiten der Schallplatte besondere Affinitäten? Welche Medien bieten sich zu einem Verbund mit der Schallplatte besonders an?

$\mathrm{Zu}$ den einsinnigen skriptiven und visuellen Medien besteht von seiten der Schallplatte die Affinität der Ergänzung. Die Schallplatte ist das Komplimentär-Medium für Texte und Bilder. ${ }^{20}$ Durch diese Bestimmung der Schallplatte werden ihre bisherigen Funktionen (Einstieg und Einsicht) wesentlich verbreitert, nämlich sie kann zum Lehr- und Lernmittel werden nicht nur auf den Lernstufen ${ }^{27}$ der Motivation und der Einsicht in eine Lösung, sondern in ähnlicher Weise auch auf den Lernstufen 
des Ausführens, des Verfestigens und der Integration des Gelernten. So kann z. B. in allen textorientierten Lernabläufen die Sprachplatte über den dramaturgischen Stimulus hinaus als aktuelle Dokumentation oder authentische Ergänzung ${ }^{28}$ eingesetzt werden. Vor allem können durch ein geplantes Einschieben des Mediums Schallplatte die direkten (frontalen) Aktionsformen des Lehrenden erweitert werden durch die indirekten Formen, in denen die Lernenden durch Einzel- oder (arbeitsgleiche/arbeitsteilige) Gruppenarbeit noch undifferenzierte Gesamtzusammenhänge durchsichtig zu machen versuchen.

Eindeutiger kommt das Moment der Ergänzung durch auditive Medien zum Zuge bei der Arbeit mit visuellen Medien. Angestoßen von französischen ${ }^{29}$, italienischen ${ }^{30}$ und niederländischen ${ }^{31}$ Vorbildern werden seit zwei bis drei Jahren Fotos in zunehmendem $\mathrm{Maß}$ in die Lernprozesse eingeplant. Eigene Fotohefte oder beigefügte Großfotos werden als Belege der Wirklichkeit herangezogen. ${ }^{32}$ Hier nun kann sich die Schallplatte als ergänzendes Medium zum Bild bewähren: sie führt medial eine komplementäre Dimension der Wirklichkeit ein. Ahnlich wie Lernverfahren mit visuellen Medien die Bilder zum Sprechen bringen, so ${ }_{n}$ stimulieren und aktivieren Hörbilder die Vorstellung des Hörers, weil sie ihn zwingen, zu sehen, was er hört" ${ }^{\text {"33. }}$

Nicht nur die einsinnigen, sondern auch die zweisinnigen, audiovisuellen Medien bieten sich für einen Verbund mit der Schallplatte an, und zwar vorab zu einem Verbund des Kontrastes. Denn die Audiovision ist eine neue Sprach- und Kommunikationsform, die mehr ist als die bloße Summe von synchronen Bild- und Tonaufzeichnungen; sie verbindet die Elemente Wort, Klang und Bild zu einer neuen, der Sprache verwandten Artikulationsform. Darum spricht diese neue Sprache der Audiovision heftiger und nachhaltiger die sinnenhafte Ebene des Menschen an. ${ }^{34} \mathrm{An}$ stelle des abstrakten, intellektuellen Arguments tritt die audiovisuelle Dokumentation. In dieser Umstrukturierung der allgemeinen Wahrnehmungs- und Erkenntnisformen ${ }^{35}$ kommt (ohne jeden kulturpessimistischen Akzent) den auditiven Medien eine besondere Rolle zu: sie haben auf die Mehrdeutigkeit der audiovisuellen Medien (Tonbildreihe, Film, Fernsehen) hinzuweisen und durch die größere Eindeutigkeit sprachlicher Informationen mögliche Deutungs- und Entscheidungsprozesse einzuleiten. So kann z. B. dem offenen Kurzfilm "Die Nashörner" von Jan Lenica nach E. Ionesco als Kontrastmedium die Schallplatte „Christine ${ }^{4}$, Erzählung von M. L. Kaschnitz ${ }^{36}$, gegenübergestellt werden; oder die Differenz zwischen Medium und Medium wird ausgewiesen an der Aufzeichnung des Fernsehfilms "Jesus - ein wahrer Hippie" von $H$. von Lojewski und der Tondokumentation „Jesus People - Auf der Suche nach der verlorenen Dimension".

Endlich kommt der Schallplatte im Medienverbund eine medienkritiscbe Funktion zu. In dem Maß, in dem weite Bereiche gegenwärtiger Welterfahrung als über technische Medien vermittelte Erfahrungaufgenommen wird, steigt die Notwendigkeit einer Medienkontrolle bzw. Medienkritik. ${ }^{37}$ Die auditiven Medien bilden hier nicht nur ein Gegengewicht gegen eine visuelle Überflutung, sondern fördern den kritischen Rezipienten. Denn die auditiven Medien respektieren in ihrer günstigen Belance von Distanz und Engagement im Vermittlungsprozeß die Meinung des Empfängers und lassen dadurch die jedem Medium inhärente Manipulation erkennen. So kann der spezielle Umgang mit auditiven Medien in Lernprozessen das allgemeine Selektionsverhalten ${ }^{38}$ der Rezipienten formen.

Das bisher Gesagte versteht sich nicht als ein (anachronistisches) Plädoyer für ein 
bedeutungslos gewordenes Medium, sondern gründet eigentlich auf der Überlegung, daß man nicht ohne Not das Sortiment der kommunikativen Medien verkleinern soll. Die Einsatzfelder der Schallplatte sind so weit gestreut, die Verwendungsformen sind so vielfältig, die Vorteile so zahlreich, daß in absehbarer Zeit nicht mit dem Verschwinden der Schallplatte gerechnet werden muß. Aber die „kommunikative Kompetenz" der Schallplatte wird stehen und fallen mit der Phantasie ihrer Hörer.

\section{Anmerkungen:}

1. Kommunikation wird hier sozialpsychologisch (im Anschluß an C. I. Hovland) verstanden als Ubermittlungsprozeß verbaler und nonverbaler Symbole zwischen Individuen; diese Definition setzt sich einerseits gegen G. Maletzke (Psychologie der Massenkommunikation, Hamburg 1963), andererseits gegen D. Baacke (Kommunikation als System und Kompetenz, in: „Neue Sammlung " 11 [1971] 566-580 und 12 [1972] 67-69) und K.-H. Schäfer/K. Schaller (Kritische Erziehungswissenschaft und kommunikative Didaktik, Heidelberg 1971) ab. - Einschränkend ist zu sagen, daß hier die Bedeutung der Schallplatte nicht in allen Kommunikationsprozessen erörtert wird, sondern nur in dem Sektor ,Lerngeschehen in Schule und Erwachsenenbildung'. Auf einige allgemeine Kommunikationsund Sozialisationsfunktionen der Schallplatte als Musikkonserve macht R. Reichardt: Die Schallplatte als kulturelles und ökonomisches Phänomen, Zürich 1962, S. 53 ff. aufmerksam.

2. Vgl. zur allgemeinen Einführung in die Didaktik-Diskussion und ihre bildungs-, informations- und lerntheoretischen Schulen: H. Blankertz: Theorien und Modelle der Didaktik. München 1969; außerdem F. Achtenhagen/H. L. Meyer (Hrsg.): Curriculumrevision - Möglichkeiten und Grenzen. München 1971; K. Frey: Theorien des Curriculums. Weinheim 1971; H. L. Meyer: Einführung in die Curriculum-Methodologie. München 1972.

3. Vgl. die Einzeldarstellungen: H. Heinrichs: Schulfernsehdidaktik. Bochum 1970. Sonderheft "Film, Bild, Ton" 1969 zur Hörspieldidaktik; A. A. Steiner (Hrsg.): Massenmedien in Unterricht und Erziehung. Frankfurt 1969.

4. A. Silbermann: Das Verhältnis von Umweltgestaltung und Massenmedien, in: „Universitas" 26 (1972) 853-857 macht auf die falsche Vorstellung aufmerksam, nals ob in der BRD über die Bedeutung und Wirkkraft der Massenkommunikationsmedien hinreichend Klarheit bestände ${ }^{\text {" }}$ (856). Eine Ubersicht über bisherige Forschungsergebnisse liefern F. Dröge/R. Weißenborn/H. Haft: Wirkungen der Massenkommunikation. München 1969.

5. Z. B. Entwicklungspolitik am Beispiel Tansanias, hrsg. von Rostra Theologia, Gelnhausen-Berlin 1972; Der Sieg heißt Friede, hrsg. von P. F. Bock, München 1972.

6. Von der steigenden Nachfrage nach technischen Lehrmitteln angestoßen, haben sich „Systemgemeinschaften “ von pädagogischen Verlagen und Elektrofirmen gebildet (Klett/ Bosch, Westermann/Philips), um allseitig perfekte audiovisuelle Lehr- und Lernsysteme zu produzieren. Doch die angebotenen Ausrüstungen und Programme sind von ihren Bedienungsschwierigkeiten und Anschaffungskosten her so fernab von der Schulwirklichkeit konzipiert, daß ein Berichterstatter anstatt von Didacta '72 von Utopia '72 sprach. In zunehmendem Maß wird audh auf die Gefahr einer „Bildungsindustrie ${ }^{\alpha}$ hingewiesen, die Curricula produziert, um, mehr und mehr losgelöst von pädagogischen und öffentlichen Interessen, Anteile am Lehrmittelmarkt zu sichern; vgl. G. Eigler: Auf dem Weg zu einer audio-visuellen Schule. München 1971, S. 7.

7. Vgl. die Buchreihe E. Meyer/E. F. Rother (Hrsg.): Audiovisuelle Mittler in der Unterridhtspraxis. München 1969 ff.; B. Merten/N. Scholl/A. Täubl: Medienkatalog glauben leben - handeln. München 1972; Ev. Konferenz für Kommunikation (Hrsg.): Medienkatalog 1972/73; AV-Material zu Themen aus Kirche und Gesellschaft. Frankfurt 1972. 
8. Auf diese Mängel macht besonders F. Olscewski: Technische Medien und Medienverbund in der Erwachsenenbildung, in: „Erwachsenenbildung“ 17 (1971) 1-12 aufmerksam; erste empirische Untersuchungen über Wirkungen der Veranschaulichung in Lernprozessen legten H. Düker/R. Tausch vor, in: Pädagogische Psychologie (Hrsg. F. Weinert). Köln 1967, 201-215; vgl. auch Dröge/Weißenborn/Haft, a.a.O. 110 ff.

9. Wichtige Uberlegungen in diese Richtung stellt F. Doedens an: Medien im Unterricht als didaktisches Problem, in: ${ }_{n} \mathrm{ru}^{\star} 2$ (1972) 58-62; er unterscheidet die Medien nach ihrer didaktischen Leistung: Medien zur Erhebung des Vorwissens, zur Motivation, zur Information, zur Beurteilung von Problemen, zur Planung und Durchführung von Aktionen und endlich Medien zur Effektivitätskontrolle. Vgl. auch K. W. Döring (Hrsg.): Unterricht mit Lehr- und Lernmittel. Weinheim 1971.

9.* H. T. Jüchter: Programmierte Erwachsenenbildung, Braunschweig 1970, S. 102, zählt zu den auditiven Medien:

\section{- Rundfunk, Fernsprechfunk}

- Schallplatte, Tonband

- Sprachlabor, Lernstudio.

$\mathrm{Zu}$ Schallplatte und Tonband müßte heute wohl hinzugefügt werden: der Kassettenrecorder. - Allerdings fehlen noch präzise Untersuchungsreihen über Leistungssteigerungen durch auditive Medien vergleichbar den Arbeiten von P. Strittmatter zu Tonbildreihen; vgl. AVA-Bericht, Bd. 4, München 1971.

10. Auf diese beiden (einzigen) Funktionen der Schallplatte macht H. Beddig: Film, Bild, Ton in der Erwachsenenbildung, methodische Anregungen und Hinweise, in: "Erwachsenenbildung" 14 (1968) 195-214, hier $202 \mathrm{ff}$., besonders aufmerksam; ähnlich auch K.-H. Boosmann: Die Schallplatte im Religionsunterricht der Berufsschule, in: F. Laubscher/J. Fischer (Hrsg.): Die Schallplatte in Kirche und Schule. Freiburg 1969, 15-21, hier 21.

11. Auf ein besonders geglücktes Unterrichtsmodell mit Schallplatteneinsätzen sei hingewiesen: H.-D. Müller/H. Blessenohl: Glückliche Menschen - sinnerfülltes Leben. Münster 1972.

12. Vgl. dazu den „Bielefelder" Sprechplattenkatalog '72/73. Bielefeld 1972.

13. Eine Durchsicht der verschiedenen Medienkataloge belegt diese Aussagen. Teilweise sind allerdings die beiden auditiven Formen kaum verändert von der Schallplatte auf das Tonband übergewechselt.

14. T. Jüchter, a.a.O., S. 22 macht die Unterscheidung: Lehrmittel - zur Unterstützung der Lehraktionen, Lernmittel - zum Gebrauch des Lernenden im Lernprozeß; H. Holstein unterscheidet $\mathrm{zwischen} \mathrm{Arbeitsmittel} \mathrm{und} \mathrm{Selbstbildungsmittel,} \mathrm{vgl.} \mathrm{„Pädagogische} \mathrm{Rund-}$ schau" 17 (1963), S. 531-538.

15. An Versuchen in der genannten Richtung sind vorab die Serien zu nennen: Baumann/ Beck/Wiesehöfer/Fischer: Krise und Chance - Glauben heute, München-Stuttgart 1970 [auch als Tonbänder angeboten]; Katholische Glaubensinformation Frankfurt/Main (Hrsg.): Glaube im Gespräch. Frankfurt 1971; A. Exeler/E. Walter/G. Bitter: Wort zur Antwort, Gottesfrage heute, Kirche und Gesellschaft, Gemeinde heute - Kirche morgen. Freiburg $1969 \mathrm{ff}$.

16. Vgl. Telefoninterview Freudenstein/Heinrichs, in: „Erziehungswissenschaft" 2, hrsg. W. Klafki u. a., Frankfurt 1970, 214 ff.; G. Bitter, Technische Hilfsmittel in der theologischen Erwachsenenbildung, in: A. Exeler/D. Emeis: Reflektierter Glaube. Freiburg ${ }^{2} 1972$, S. 208-221, hier S. $208 \mathrm{f}$.

17. Vgl. F. Pöggeler: Methoden der EB. Freiburg 1964; H. Tietgens: Lernen mit Erwachsenen. Braunschweig 1967; Exeler/Emeis: a.a.O., S. 133-238; H. T. Jüchter: a.a.O.; D. Emeis: Uberlegungen zur Methodenfrage in der theologischen Erwachsenenbildung, in: „Erwachsenenbildung “ 16 (1970), S. 103-118.

18. So ist es wohl kein Zufall, wenn in zwei renomierten Veröffentlichungen zur Mediendidaktik die Schallplatte gar nicht mehr behandelt wird: H. Heinrichs: Brennpunkte neuzeitlicher Didaktik. Bochum ${ }^{12} 1970 ; \mathrm{K}$. W. Döring: a.a.O.

19. Vgl. H. T. Jüchter: a.a.O., S. 88. 
20. H. T. Jüchter: ebd.

21. Vgl. R. Reichardt: a.a.O., S. 53 f.

22. Vgl. P. Babin (Hrsg.): Audiovisuelle Glaubenserziehung. Köln 1972, S. 174 ff. und G. Bitter, Art. Bild, in: Exeler/Scherer: Glaubensinformation. Freiburg ${ }^{3} 1972$, S. $47 \mathrm{ff}$.

23. Th. W. Adorno (Prolog zum Fernsehen, in: Eingriffe, Frankfurt ${ }^{8} 1970$, S. 69-80) warnt vor den Stereotypen des Fernsehens: „Das Starre wird nicht aufgelöst, sondern verhärtet. Die Vokabeln der Bilderschrift sind Stereotypen" (78). Vgl. auch W. van Appeldorn: Die optische Revolution. Reinbek 1972.

24. W. Nowak: Visuelle Bildung, Ein Beitrag zur Didaktik der Film- und Fernseherziehung. Villingen 1967; ders.: Art. Visuelle Bildung, in: H. Heinrichs (Hrsg.): Lexikon der audiovisuellen Bildungsmittel. München 1971, S. $335 \mathrm{ff}$.

25. Vgl. oben S. 2 f. Die Medienfrage - welchem Medium ist im Lernprozeß welche Funktion zugewiesen? - stellt sich also im Anschluß an die Operationalisierung der Lernziele und die Wahl der adäquaten Lernverfahren. - $H$. Gutschow: Die Bedeutung der Unterrichtsmittel für den Englischunterricht, in: K. H. Döring, a.a.O. 267-276 macht auf die Steigerung der Lerneffizienz durch Medienverbund aufmerksam.

26. Diese Bestimmung gilt allerdings nicht ausschließlich für die Schallplatte, sondern allgemein für die auditiven Medien, zumindest für Tonband und Hörfunk.

27. Vgl. dazu das Lernstufenmodell von H. Roth: Pädagogische Psychologie des Lehrens und Lernens. Hannover ${ }^{10} 1967$, S. 248.

28. Vgl. z. B. die Reihen der Deutschen Grammophon Gesellschaft: „Literarisches Archiv“, "Paperback" und "Hörspiel heute".

29. Vgl. P. Babin: a.a.O. passim; dgl. P. Babin u. a.: Fotosprache, Der Mensch - Bilder. Gelnhausen 1971.

30. Don Mazzi: Die Botschaft Jesu im Isolotto, Mainz 1969; in der Originalausgabe bestehen die Arbeitsblätter der Schüler aus Großfotos und knappen Texten.

31. Koenen/Thuring: Christsein im Alltag. München 1968.

32. Vgl. die von G. Kiefel herausgegebenen Fotohefte: Hoffnung für heute und morgen. Gladbeck 1969; Wir suchen das Glück. Wuppertal 1970; Auf den Spuren des Glücks. Wuppertal 1971 und die Arbeitsmappe für Schüler von Oser/Germann/Kirchhofer: Salz der Erde. Olten 1970.

33. G. Sieg, Lehr- und Lernmittel im Religionsunterricht, Vorbemerkungen zu einer Theorie der didaktischen Medien, in: D. Zilleßen (Hrsg.): Religionsunterricht und Gesellschaft. Düsseldorf 1970, S. 113-125, hier S. 122; G. Sieg verweist auf McLuhan, der das "Hörbild“ zu den visuellen Medien zählt, während er das Fernsehen für ein „taktiles" Medium hält. Vgl. M. McLuhan/Qu. Fiore: Das Medium ist Massage (Ullstein TB 3639). Berlin 1969, S. 117 und J. Miller: M. McLuhan (dtv 825). München 1962.

34. M. McLuhan/Qu. Fiore nennen die Audiovision die Sprache der „Vereinigung und Beteiligung"; "die einst anerzogene Fähigkeit distanzierten Beobachtens ist in dieser neuen Zeit (der Audiovision) völlig belanglos geworden" (a.a.O., S. 8); vgl. auch P. Babin: a.a.O., S. 26 u. ö. und Dröge/Weißenborn/Haft, a.a.O., S. 112.

35. Vgl. zur zunehmenden Bedeutung der Empirie für die allgemeine Erkenntnis die TheoriePraxis-Diskussion.

36. Als Sprechplatte bei Christophorus, Freiburg CLX 75517 erschienen. - Vielleicht ist aber auch auf eine Gegenstimme hinzuweisen: K. Meissner: Die Dritte Aufklärung, Wissenschaft und Erwachsenenbildung in einer Informationsgesellschaft. Braunschweig 1969, S. 155: „Das Bild gerät an die Grenze der Sagbarkeit und kann kaum durch das Wort wieder eingefangen werden. Es hat sich ein eigener Bereich des Bildes erschlossen, Bildersprache, die das gesprochene oder geschriebene Wort nur noch kommentieren kann." Beachtenswert angesichts des zunehmenden Sprachverlustes in der Kulturindustrie ist die Wicderentdeckung der Sprache in der Literatur (G. Wellersholt, P. Handke, E. Jandl), in den Sprachwissenschaften, in der Philosophie und Theologie.

37. W. Menningen, Fernsehen - Unterhaltungsindustrie oder Bildungsinstitut. Stuttgart 1971, S. 69-73.

38. Vgl. dazu P. Atteslander: Informationsflut und Informationsdefizit in unserer Uberflußgesellschaft, in: „Universitas“ 26 (1971) 159-168. 


\section{S UM M A R Y}

The record has been somewhat neglected as a Communications medium in Communications theory. From the didactic, curriculum research, and especially from pedagogical experience it has once more come under consideration. We have here the particular media qualifications which are of interest to the man in the work of teaching, using records within the multimedia system. Bitter concludes that the record is coming into its own, especially as it complements both written and visual media.

\section{R É SUM É}

Le disque comme moyen de communication a été quelque peu négligé dans la théorie publicistique. Il fut continuellement remis en question du point de vue de la didactique, de la recherche et surtout de la pratique en pédagogie. Et ici, ce sont surtout les caractères spécifiques qui intéressent le practicien dans l'ensemble de l'utilisation du disque en liaison avec les autres moyens. Avec amertume, on arrive à la conclusion qu'ici le disque a aussi sa chance, car le disque possède l'affinité de compléter les autres moyens.

\section{RESUMEN}

La teoría publicista desatendió al disco como medio de comunicación social. La didáctica, la investigación del curriculum y sobre todo la práctica pedagógica le han hecho de nueco objeto de polémica constructiva. Sus propiedades específicas como instrumento de comunicación social son las que ante todo interesan a los pragmáticos para su utilización en el mundo de la comunicación. Bitter llega a la conclusión de que, también en el futuro, el disco tiene un lugar en dicho mundo, pues, respecto de los medios de comunicación predominantemente visuales, el disco tiene la afinidad del complemento. 